\section{Arreflexia pós-calórica bilateral: aplicabilidade clínica da reabilitação vestibular}

\author{
Roseli Saraiva Moreira Bittar ${ }^{1}$, \\ Marco Aurélio Bottino ${ }^{1}$, Maria Elisabete Bovino \\ Pedaliní ${ }^{2}$, Jeanne da Rosa Oiticica Ramalho ${ }^{3}$, \\ Camila de Giacomo Carneiro ${ }^{4}$
}

\section{Bilateral vestibular loss after caloric irrigation: clinical aplication of vestibular rehabilitation}

\section{Resumo / Summary}

Palavras-chave: arreflexia pós-calórica, reabilitação vestibular, equilíbrio. Key words: bilateral vestibular loss, vestibular rehabilitation, balance.

\begin{abstract}
$\mathbf{A}$ perda bilateral da função vestibular é rara em pacientes com vertigem e desequilíbrio, porém os sintomas muitas vezes são incapacitantes e seu tratamento é tema controverso na literatura. Objetivo: Nosso objetivo foi avaliar e descrever a resposta clínica de pacientes com distúrbio do equilîbrio corporal secundário à arreflexia vestibular pós-calórica bilateral, documentada pela eletronistagmografia, submetidos à Reabilitação Vestibular. Forma de estudo: Retrospectivo, inclui um desenho de descrição de casos. Método: Foram avaliadas as respostas de 8 pacientes portadores de arreflexia pós-calórica bilateral submetidos à Reabilitação Vestibular, observando-se a relação entre os resultados de exame e sintomas pré e pós-tratamento. A avaliação da resposta clínica foi feita por meio de escala analógicovisual. Resultados: Após a Reabilitação Vestibular, 7 (87,5\%) dos 8 pacientes submetidos à terapia apresentaram melhora clínica. Conclusão: embora não seja esperada melhora completa do equilîbrio corporal, a Reabilitação Vestibular é uma terapia eficaz na recuperação desses pacientes.
\end{abstract}

\begin{abstract}
B ilateral vestibular loss is a rare diagnosis among patients with dizziness and imbalance. Nevertheless, symptoms are often disabling and therapy is yet to be establish. Aim: To evaluate and describe the clinical outcome of patients with imbalance due to bilateral vestibular loss after caloric test, treated with An analog visual scale was used to evaluated clinical results. Vestibular Rehabilitation. Study design: Retrospective case report. Method: Pre and post treatment outcomes were evaluated in 8 individuals suffering from post caloric bilateral vestibular paresis whose were submitted to vestibular rehabilitation. Results: After Vestibular Rehabilitation, $7(87,5 \%)$ of 8 patients had clinical improvement. Conclusion: Although is not expected entirely compensation for bilateral vestibular loss, the vestibular rehabilitation may be use as a therapeutic method for these patients.
\end{abstract}

\footnotetext{
${ }^{1}$ Assistente Doutor do Setor de Otoneurologia do HCFMUSP.

${ }^{2}$ Fonoaudióloga Responsável pelo Ambulatório de Reabilitação Vestibular do HCFMUSP

${ }^{3}$ Pós-Graduando - Nível Doutorado da Disciplina de Otorrinolaringologia da FMUSP.

${ }^{4}$ Médico Estagiário do Setor de Otoneurologia do HCFMUSP.

Disciplina de Otorrinolaringologia do Hospital das Clínicas da FMUSP - Serviço do Prof. Aroldo Miniti

Endereço para Correspondência: Roseli Saraiva Moreira Bittar / Depto de ORL do HCFMUSP -

R. Dr. Enéas de Carvalho Aguiar, $2556^{\circ}$ andar sala 6021 05403-000 São Paulo SP Brasil E-mail: otoneuro@hcnet.usp.br

Artigo recebido em 24 de setembro de 2003. Artigo aceito em 30 de outubro de 2003.
} 


\section{INTRODUÇÃO}

A perda vestibular bilateral ou arreflexia vestibular bilateral (AVB) pode ser definida como total ausência de resposta do sistema vestibular aos estímulos provocativos, diagnosticada pela eletronistagmografia (ENG) ou pela prova rotatória pendular decrescente (PRPD).

A perda bilateral da função vestibular periférica é rara em pacientes com queixa de vertigem e desequilíbrio, e representa $0,6 \%$ das eletronistagmografias ${ }^{1}$. As prováveis causas da AVB estão relacionadas ao uso de ototóxicos, traumas, meningite, infecção labiríntica, tumores bilaterais (schwanoma vestibular), otosclerose, hidropisia endolinfática, cirurgia otológica, doença auto-imune e idiopática ${ }^{2,3}$. Dentre todas as etiologias citadas, a mais freqüente é a ototoxicidade, responsável por $21 \%$ dos casos ${ }^{1}$.

Os principais sintomas descritos na AVB incluem a oscilopsia e o desequilíbrio. O primeiro é a expressão clínica do comprometimento do reflexo vestíbulo-ocular (RVO), e é caracterizado pela oscilação da imagem, que se intensifica durante os movimentos cefálicos e a marcha. Os objetos à distância parecem turvos e distorcidos, situação que reduz a capacidade de aproveitamento das referências visuais para manutenção do equilibrio. Já o desequilỉbrio, decorrente do comprometimento do reflexo vestíbulo-espinal (RVE), torna-se intenso em ambientes escuros e/ou superfícies irregulares, uma vez que são necessários pelo menos dois sistemas de informação para que o equilíbrio corporal seja mantido, e na ausência da informação vestibular, as informações visuais e proprioceptivas são utilizadas em conjunto. Por estes motivos, nesse grupo de pacientes há uma maior incidência de quedas, $51 \%$ na AVB contra $30 \%$ nas perdas vestibulares unilaterais, independente da faixa etária ${ }^{4,5}$.

A recuperação funcional desses pacientes pode ser obtida de forma parcial pela reabilitação vestibular (RV), que tem os seguintes objetivos: melhorar a estabilidade postural e o equilíbrio, principalmente durante a deambulação.

Dentro dos programas de RV, existem duas principais estratégias para trabalhar a plasticidade neuronal: a adaptação vestibular e a substituição vestibular. Essa distinção é importante pois o tipo de tratamento adotado está relacionado ao distúrbio do equilíbrio apresentado pelo paciente. A primeira estratégia, conhecida como adaptação vestibular, está indicada aos pacientes que possuem função vestibular remanescente e procura resgatar qualquer vestígio de informação residual. Baseia-se nas mudanças que ocorrem em longo prazo no SNC em decorrência de respostas do sistema vestibular às novas informações sensoriais recebidas.

A adaptação vestibularé baseada principalmente no estímulo chamado "retinal slip", ou "sinal de erro", que é o deslizamento das imagens sobre a retina conseqüente à falência do RVO, cuja função é manter a imagem fixa na retina durante os movimentos cefálicos. A presença do "sinal de erro", evoca respostas do Sistema Nervoso Central (SNC) que são capazes de modificar as características do RVO. Essa estratégia de tratamento visa portanto, a mudança do ganho, fase, ou direção da resposta vestibular ${ }^{3}$.

A outra estratégia, conhecida como substituição vestibular, procura trabalhar mecanismos alternativos para suprir a perda da função vestibular. Dentre eles, a substituição sensorial (reflexo cervico-ocular), motricidade ocular (sacadas), e a predição ou antecipação ${ }^{3}$. O paciente é encorajado a confiar nas informações proprioceptivas e visuais, para estabilizar a fixação ocular e manter a postura na ausência das aferências vestibulares. As dicas visuais e somatossensoriais são usadas em conjunto com eventuais informações vestibulares residuais, modificando a programação prévia existente no SNC, com conseqüente melhora da postura e da marcha. Estas estratégias compensatórias são úteis em situações em que o equilíbrio corporal é intensamente requisitado e estão indicadas para casos de deaferentação vestibular bilateral.

Alguns autores comprovaram a efetividade da RV no tratamento de pacientes com perda vestibular bilateral ${ }^{4,6}$, enquanto outros discordam deste fato $^{7,8}$.

\section{OBJETIVO}

O objetivo deste trabalho é avaliar e descrever a resposta clínica de pacientes com distúrbio do equilíbrio corporal secundário à arreflexia vestibular pós-calórica bilateral, documentada pela eletronistagmografia, submetidos à Reabilitação Vestibular.

\section{CASUÍSTICA E MÉTODO}

Este estudo seguiu todas as normas vigentes no Hospital das Clínicas da FMUSP, determinadas pela Comissão de Ética para Análise de Projetos de Pesquisa. É um estudo retrospectivo descritivo, dos pacientes atendidos no Setor de Otoneurologia, da Disciplina de Otorrinolaringologia da FMUSP, que apresentavam distúrbio do equilíbrio corporal associado à perda vestibular pós-calórica bilateral.

Foram incluídos os pacientes com distúrbio do equilíbrio corporal por AVB, submetidos a tratamento com RV. O diagnóstico foi firmado através de eletronistagmografia, definido como ausência completa de resposta à estimulação nas provas calóricas $\left(44^{\circ}-30^{\circ}-18^{\circ} \mathrm{C}\right.$ ).

Todos os pacientes foram submetidos à avaliação otoneurológica clássica que inclui história clínica, exame otorrinolaringológico, exame dos pares cranianos, provas de equilibrio e cerebelares, eletronistagmografia, conforme rotina instituída no Ambulatório de Otoneurologia do HCFMUSP. Em alguns casos foi realizada a posturografia dinâmica computadorizada (PDC) e a prova rotatória pendular decrescente (PRPD), que não constituíram critérios de seleção para esta avaliação. Para análise do resultado da PDC foi considerado o Teste de Integração Sensorial (TIS) por meio da Análise Sensorial (AS). 
Os pacientes foram então submetidos à RV, que incluiu exercícios de substituição, baseados no protocolo de Cawthorne\&Cooksey modificado, associados a exercícios complementares, enfatizando o treinamento do equilíbrio estático e dinâmico ${ }^{9}$. As terapias foram adaptadas conforme necessidade individual, realizadas no ambulatório, e os pacientes foram orientados a executar os exercícios em domicílio, retornando para reavaliação quinzenalmente.

Foram consideradas variáveis do estudo idade, sexo, sintomas pré e pós-tratamento, e o diagnóstico clínico. A avaliação da resposta clínica ao tratamento foi feita por meio de uma escala analógico-visual: remissão ( $\mathrm{R}$ - 100\%), melhora parcial (MP - entre 50\% e 90\%), sem melhora (SM abaixo de 50\%). A análise estatística inclui um desenho de descrição de casos.

\section{RESULTADOS}

A amostra constitui-se de 8 casos de arreflexia vestibular pós-calórica bilateral, 4 pacientes do sexo feminino e 4 do sexo masculino, com idade que variou de 33 a 87 anos (média de 57,6 e desvio padrão de 20,1). O diagnóstico etiológico e a idade dos diversos pacientes podem ser observados na Tabela 1.

Os sintomas mais encontrados foram desequilíbrio $(87,5 \%)$, vertigem $(87,5 \%)$, instabilidade $(75 \%)$. A história de quedas foi referida por $37,5 \%$ dos pacientes. Observou- se comprometimento da marcha em dois casos (25\%) e oscilopsia em dois casos (25\%), conforme distribuição representada na Tabela 2.

O tempo de RV não variou entre os 8 casos e a duração média foi de 3 meses ( 6 a 7 sessões de retorno). Os resultados do tratamento pela RV estão expostos no Gráfico 1. A melhora clínica ocorreu em 7 (87,5\%) dos 8 pacientes submetidos à terapia.

Dos pacientes estudados, 2 foram submetidos a PRPD e 3 realizaram PDC. A PRPD mostrou ausência de resposta ao estímulo rotacional no paciente 3 e reflexos intensamente reduzidos no paciente 8 . Com relação a PDC, foi considerada a resposta da análise sensorial (AS) no teste de integração sensorial (TIS) para avaliação da função vestibular, que esteve presente no exame inicial apenas do paciente 6. Após a RV foi documentada a melhora do padrão vestibular mesmo no paciente 3, que não apresentou resposta vestibular à primeira observação. Observou-se ainda a melhora dos padrões visual e somatossensorial nos 3 pacientes. Os dados obtidos antes e após a RV podem ser observados na Figura 1.

\section{DISCUSSÃO}

A ausência completa de resposta na prova calórica e ou na prova rotatória pendular decrescente é achado raro em pacientes com queixa de vertigem e desequilíbrio ${ }^{1,10}$. Essa baixa freqüência justifica a escassez de literatura sobre

Tabela 1. Representação dos Pacientes e das Etiologias da Arreflexia Vestibular.

\begin{tabular}{cccccccc}
\hline Paciente & SEXO & Idade & Iniciais & Diagnóstico & PRPD & PDC & Resposta RV \\
\hline 1 & F & 51 & JRM & ototoxicidade & NR & NR & remissão \\
2 & M & 87 & NJ F & IVB & NR & NR & sem melhora \\
3 & F & 44 & MCR & idiopática & sem resposta & alterada & melhora \\
4 & M & 54 & J MS & TCE & NR & NR & melhora \\
5 & F & 65 & DMA & ototoxicidade & NR & NR & melhora \\
6 & M & 41 & ACTA & idiopática & NR & alterada & melhora \\
7 & M & 33 & FJC & TCE & NR & NR & melhora \\
8 & F & 86 & VMPM & IVB & hiporreflexiasevera alterada & melhora \\
\hline
\end{tabular}

IVB = insuficiência vértebro-basilar; TCE = traumatismo crânio-encefálico;

$\mathrm{NR}=$ não realizada; $\mathrm{PRPD}=$ prova rotatória pendular decrescente;

PDC $=$ posturografia dinâmica computadorizada.

Tabela 2. Distribuição dos Sintomas nos Pacientes com Arreflexia Vestibular Bilateral.

\begin{tabular}{|c|c|c|c|c|c|c|c|}
\hline Paciente & iniciais & oscilopsia & desequilíbrio & vertigem & instabilidade & queda & alteração de marcha \\
\hline 1 & JRM & - & + & + & - & - & - \\
\hline 2 & NJ F & - & + & - & + & - & + \\
\hline 3 & MCR & + & + & + & + & + & - \\
\hline 4 & J MS & - & + & + & + & + & - \\
\hline 5 & DMA & - & + & + & + & - & - \\
\hline 6 & ACTA & - & - & + & - & - & - \\
\hline 7 & FJ C & - & + & + & + & - & + \\
\hline 8 & VMPM & + & + & + & + & + & - \\
\hline
\end{tabular}

(+): sintoma presente; (-): sintoma ausente. 


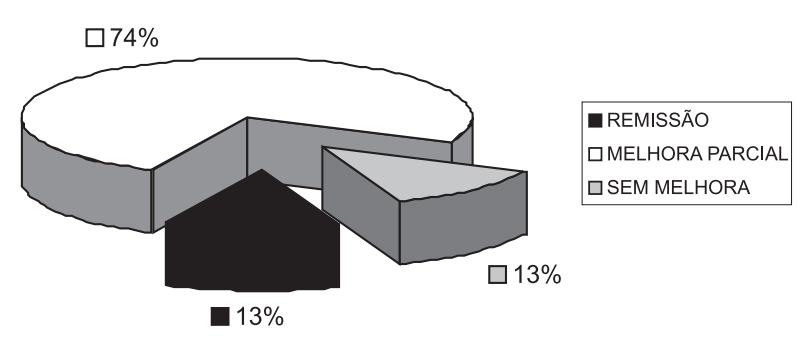

Gráfico 1. Distribuição dos pacientes segundo a melhora clínica apresentada após RV.

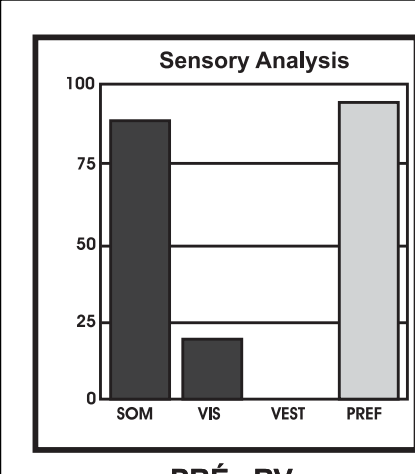

PRÉ RV

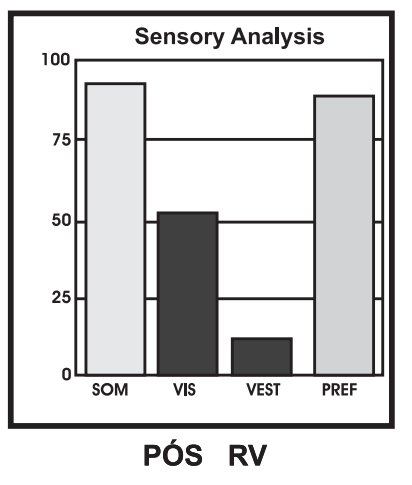

Figura 1. Análise Sensorial da paciente 3 antes e após o programa de RV.

SOM = função somatossensorial; VIS = função visual; VEST = função vestibular; PREF = preferência visual.

Obs: As barras escuras estão abaixo do padrão de normalidade para a idade.

o assunto, o tamanho da nossa amostra, e a dificuldade de se conduzir ensaios clínicos prospectivos. Um outro fator restritivo é a dificuldade de comparação entre os resultados dos diversos autores, pois os critérios diagnósticos utilizados são díspares.

A oscilopsia esteve presente em $25 \%$ dos casos, e não foi um sintoma tão prevalente. Este fato concorda com outros achados na literatura ${ }^{1,7,8}$. Porém, assim como os demais, este estudo é retrospectivo, e o sintoma pode não ter sido investigado por ocasião da coleta da história clínica. O mesmo pode-se dizer em relação às quedas, encontradas em $37,5 \%$ dos casos, contrastando com dados de literatura, que descrevem incidência de quedas em $70 \%$ das perdas vestibulares bilaterais na faixa etária inferior a 65 anos $^{5}$.

Quanto à etiologia, a literatura cita a ototoxicidade e as causas idiopáticas como as mais freqüentes ${ }^{7,8,10,11}$. Contu- do, não houve predomínio quanto ao diagnóstico etiológico na nossa amostra, provavelmente pelo tamanho pequeno da mesma.

Quando avaliamos o resultado da RV, observamos melhora clínica em $7(87,5 \%)$ dos 8 pacientes submetidos à terapia. A melhora foi descrita de forma diferenciada pelos diversos pacientes, porém todos relataram maior estabilidade no desempenho das atividades físicas habituais.

Estudando criteriosamente cada paciente, vamos discorrer agora sobre os aspectos clínicos observados individualmente. O paciente 1, que referiu remissão, fez dois meses de terapia, e ao final da mesma conseguiu abandonar a bengala. Embora mantivesse problemas de equilibrio após o tratamento, o fato de poder se locomover sem apoio foi considerado pelo doente uma grande vitória. Essa observação nos leva a concluir que, mesmo que pela nossa análise crítica o paciente não apresente um equilibrio que possa ser dito perfeito, deve ser considerada a expectativa do próprio paciente. Dessa maneira, a expectativa de resultados que pretendemos em uma criança é diversa do idoso, uma vez que o último pode ter objetivos limitados, como sair à rua sem auxílio, por exemplo.

O paciente 2 (87 anos), que não apresentou melhora dos sintomas após reabilitação, além da idade avançada não foi assíduo às sessões de retorno, embora tenha completado o programa. Neste caso cabem algumas colocações importantes como o envelhecimento, que compromete o resultado final do tratamento. Do ponto de vista funcional, a diminuição gradual do número de células ciliadas e de neurônios vestibulares compromete o ganho do RVO, e limita os movimentos de velocidade pela dificuldade de fixação da imagem na retina. O deslocamento das imagens na retina é maior, e conseqüentemente, a manutenção do foco na retina durante o movimento cefálico é pior. O déficit vestibular bilateral progressivo é uma das características do envelhecimento, e justifica o porquê das disfunções vestibulares bilaterais idiopáticas serem mais comuns nos idosos ${ }^{3,7}$. Outra manifestação do envelhecimento é a redução da capacidade de ajuste do sistema vestibular pelo SNC, e portanto, maior dificuldade de compensação e controle postural. Os déficits visuais também são comuns, e decorrem dos declínios da acuidade visual (glaucoma, catarata, degeneração macular), da percepção de profundidade, da capacidade de acomodação, e da perseguição uniforme. As alterações proprioceptivas complementam a gama de fatores limitantes à manutenção adequada do equilîbrio nos idosos, e resultam de redução da sensibilidade tátil, que associada à diminuição da força muscular, a lentificação das respostas, e a hipotensão postural (muitas vezes potencializada pelo uso de drogas hipotensoras) dificultam a recuperação da perda vestibular bilateral. Por estas limitações, pelo maior número de fatores de risco presentes, maior incidência de quedas, comorbidades associadas e redução da cognição, o idoso geralmente precisa ser tratado por um período mais longo ${ }^{3,10}$. Ainda nesse mesmo paciente, 
a segunda colocação de fundamental importância é o acompanhamento inadequado durante o trabalho de RV, expresso pela sua falta de assiduidade ao acompanhamento terapêutico. A insistência de pontos fundamentais de esclarecimento, o trabalho do aspecto cognitivo, a correção dos exercícios, que dificilmente são perfeitamente compreendidos à primeira explicação, além da participação ativa do paciente, são pontos chave para o bom resultado da RV.

Segundo nossa análise, a maioria dos casos apresentou melhora parcial com o tratamento, e esse aspecto está seguramente relacionado ao severo comprometimento das aferências vestibulares. O equilíbrio postural só é possível mediante informação de pelo menos dois sistemas e, quando há perda substancial da função de um ou mais deles, as limitações passam a ser maiores ${ }^{12}$. Essa limitação é particularmente importante quando é lesado o sistema vestibular, cuja informação é decisiva quando há presença de conflito sensorial entre dois sistemas. A recuperação decorrente da perda vestibular bilateral é mais lenta quando comparada à perda unilateral, e não mais se espera que a estabilidade postural seja completamente normal ${ }^{4}$. Os pacientes 3 e 4 relataram melhora da marcha e da habilidade para subir e descer escadas sem apoio. O paciente 5 obteve melhora da tontura e passou a atravessar a rua sem acompanhante. $\mathrm{O}$ paciente 6 refere melhor desempenho nas atividades domésticas, porém com persistência da vertigem em ambientes abertos, fato compreensível, pois não há a referência de verticalidade, que para ele funciona como apoio visual. O paciente 7 relata maior independência física, porém com persistência de desequilíbrio discreto. O paciente 8 relata melhora da tontura, porém com dificuldade ao subir e descer escadas sem apoio.

Com relação ao trabalho de RV propriamente dito, os resultados em pacientes com AVB variam bastante na literatura. Um dos fatores implicados nesses resultados parece ser a estratégia de tratamento adotada. Alguns autores relatam ausência de melhora clínica, pós-terapia de RV, em 50\% dos pacientes com $\mathrm{AVB}^{7,8,10}$. Nestes estudos, a estratégia de tratamento adotada foi baseada em exercícios de habituação, que não são os mais adequados para o tratamento das perdas vestibulares bilaterais ${ }^{4,13}$. É fácil entender que nos casos de AVB são necessários exercícios de substituição, que estimulem as vias visuais e somatossensitivas remanescentes, além dos mecanismos de predição do SNC, uma vez que não há estímulos vestibulares a serem adaptados. Outros autores relatam que pacientes que receberam tratamento com exercícios de adaptação e substituição apresentaram maior estabilidade na marcha e ao subir escadas, quando comparados com pacientes submetidos à terapia com exercícios isométricos e de condicionamento ${ }^{4,6,13}$. Esse aspecto também é facilmente compreendido porque o fortalecimento muscular não apresenta efeito marcante nas aferências vestibulares e suas conexões, pois não têm a finalidade de treinar o equilibrio.
Resta finalmente avaliar a prova calórica como exame prognóstico do resultado da RV. Por ser uma prova de estimulação de baixa freqüência, seus resultados podem ser falhos no diagnóstico final de "ausência de função vestibular", e o padrão ouro para o diagnóstico é considerado a PRPD $^{14}$.

Os movimentos do dia-a-dia possuem velocidades angulares entre 0,01 e $8 \mathrm{~Hz}$ e, teoricamente, uma disfunção vestibular atingiria apenas parte deste intervalo de freqüência. O teste mais amplamente usado na investigação das vestibulopatias é a ENG, e suas principais vantagens são o custo e a possibilidade de testar cada labirinto separadamente. Sua maior limitação é a documentação apenas do canal semicircular horizontal e das baixas freqüências de aceleração angular $(0,002$ a $0,004 \mathrm{~Hz})$, ou seja, bem abaixo da faixa fisiológica que compreende o RVO. Para avaliar as freqüências mais altas, a melhor opção é a PRPD, que permite avaliar a função vestibular bilateral, em baixas e altas freqüências $(0,1 \mathrm{a} 1 \mathrm{~Hz})$. Sua principal limitação é o custo e a dificuldade de testar freqüências além de $2 \mathrm{~Hz}$, pois nestes casos a aceleração do corpo dificulta a estabilização da cabeça, e a distinção entre componentes lentas e rápidas ${ }^{15}$.

As assimetrias vestibulares podem ser diagnosticadas com relativa precisão pela ENG, isto porque o lado comprometido pode ser comparado ao normal. Contudo, nas perdas vestibulares bilaterais, a PRPD torna-se prova importante na avaliação do RVO, pois a ausência de resposta na ENG não exclui a possibilidade de deteç̧ão de respostas em outros testes vestibulares, em que são avaliadas outras freqüênci$\mathrm{as}^{7,8,10,12}$. No entanto, essa particularidade não implica em considerar que o diagnóstico de AVB, dado pela prova calórica, deva ser desconsiderado, pois as repostas, embora presentes em outros testes, podem estar reduzidas, comprovando a disfunção vestibular. Embora não seja capaz de quantificar o déficit vestibular, a ENG é um teste relativamente sensível no diagnóstico precoce da $\mathrm{AVB}$, pois a tendência é que nestes casos a perda seja maior nas baixas freqüências ${ }^{15}$. Deve-se ainda considerar o fato de que a ENG é um instrumento amplamente utilizado na prática clínica e, por vezes, o único disponível. Considerar e avaliar seu resultado implica em fornecer dados ao especialista que the dêem condições de planejamento e avaliação do prognóstico desses pacientes. De fato, nos dois pacientes de nossa amostra que foram submetidos a PRPD, observamos melhora de mais de $50 \%$ dos sintomas, embora a prova mostrasse resposta em apenas um deles. Assim, não observamos resposta diferencial ao tratamento quando consideramos os resultados da PRPD aliados à ENG.

Quanto à PDC, podemos dizer que é um teste que quantifica a estabilidade postural em diversas condições, medindo a contribuição e a interação das diversas aferências sensoriais (visual, vestibular, e somatossensorial). É útil na avaliação objetiva do impacto funcional da perda vestibular e dos sistemas comprometidos na manutenção do equilí- 
brio. Na AVB há disfunção do RVO e do RVE, que são observadas na PDC pelo aumento da oscilação nas condições sensoriais cuja aferência visual e ou somatossensorial estão ausentes ou são conflitantes. A maior disfunção pode ser observada nas condições 5 e 6 , tipicamente vestibulares, com perda rápida do equilíbrio e tendência de uso da estratégia quadril ${ }^{2,16}$

Quando observamos a análise sensorial dos pacientes submetidos à PDC, verificamos que houve ausência de resposta vestibular em apenas dois deles (pacientes 3 e 8), que apresentavam ainda resposta visual abaixo do padrão de normalidade (Figura 1). A paciente 3, do sexo feminino, 44 anos de idade, referia oscilopsia e sua principal queixa era não poder freqüentar ambientes de grande estimulação visual, pois apresentava quedas, que também estavam presentes na paciente 8 . Podemos interpretar essas quedas como a sobrecarga de estímulos oferecida ao sistema visual, que na ausência da informação vestibular assume papel fundamental na manutenção do equilíbrio junto ao $\mathrm{SNC}^{17}$. Os pacientes portadores da AVB tendem a equilibra-se de início nas pistas visuais, e com o tempo aprendem a utilizar-se das pistas somatossensoriais ${ }^{18}$. Por essa razão, os ambientes que apresentam muita informação visual e que obrigam o indivíduo a promover rotações cefálicas, que diminuem sua referência visual, como shopping centers ou mercados, provocam desconforto ${ }^{19}$.

Na paciente 3, os dados da PDC pós-tratamento comprovam que houve não só melhora da participação vestibular, como também visual e proprioceptiva, embora apenas a função somatossensorial atingisse o padrão de resposta esperado para a idade (Figura 1). A melhora do padrão vestibular pode ser atribuída a aferências de outras regiões sensitivas ${ }^{20}$, que suprem de forma limitada a informação labiríntica faltante. Nesse aspecto, a pré-programação central é de fundamental importância e, embora a paciente apresente bons resultados a estímulos previsíveis, nos movimentos inesperados o fator surpresa é motivo para quedas ao solo. Apesar dos resultados, a paciente sentiu-se muito gratificada pois desapareceu a oscilopsia, não apresentou mais quedas, conseguiu andar de salto alto, dançar e sair à noite, atividades que não realizava antes da RV.

Já a paciente 8 apresentou melhora da função visual, anteriormente deficitária, e não houve qualquer melhora na função vestibular, observada na paciente anterior. Após a RV a paciente sentiu-se amplamente gratificada embora persistisse o desequilibrio quando ela passava de um ambiente a outro, explicada pelo conflito na referência visual. Outra dificuldade que persistiu foi subir e descer escadas sem apoio.

Os três pacientes analisados apresentaram o mesmo grau de melhora, independente da resposta à PDC e portanto, não houve resposta diferencial quando os resultados da PDC foram associados à ENG.

\section{CONCLUSÃO}

Embora não seja esperada uma completa recuperação funcional do equilîbrio, a utilização da Reabilitação Vestibular nos casos de arreflexia pós-calórica bilateral é conduta adequada e seus resultados devem ser interpretados como positivos em função das expectativas do terapeuta e do paciente.

\section{REFERÊNCIAS BIBLIOGRÁFICAS}

1. Vibert D, Liard P, Häusler R. Bilateral idiopathic loss of peripheral vestibular function with normal hearing. Acta Otolaryngol (Stockh) $1995 ; 115: 611-5$.

2. Herdman SJ, Sandusky AL, Hain TC, Zee DS, Tusa RJ. Characteristics of postural stability in patients with aminoglycoside toxicity. Jl Vest Res 1994; 4:71-80.

3. Herdman SJ. Reabilitação Vestibular. 2 $2^{\underline{a}}$ ed. São Paulo, Brasil: Editora Manole; 2002.

4. Herdman SJ, Schubert MC, Tusa RJ. Strategies for balance rehabilitation - fall risk and treatment. Ann New York Acad Sci 2001; 942: 394-412.

5. Herdman SJ, Blatt PJ, Schubert MC, Tusa RJ. Falls in patients with vestibular deficits. Am J Otol 2000; 21:847-51.

6. Krebs DE, Gill-Body KM, Riley PO. Double-bind, placebo-controlled trial of rehabilitation for bilateral vestibular hypofunction: preliminary report. Otolaryngol Head Neck Surg 1993; 109:735-41.

7. Sargent EW, Goebel JA, Hanson JM, Beck DL. Idiopathic bilateral vestibular loss. Otolaryngol Head Neck Surg 1997; 116:157-62.

8. Telian SA, Shepard NT, Smith-Wheelock M, Hoberg M. Bilateral vestibular paresis: diagnosis and treatment. Otolaryngol Head Neck Surg 1991; 104: 67-71.

9. Pedalini MEB, Bittar RSM. Reabilitação vestibular: uma proposta de trabalho. Pró-Fono 1999; 11(1):140-4.

10. Gillespie MB, Minor LB. Prognosis in bilateral vestibular hypofunction. Laryngoscope 1999; 109(1):35-41.

11. Brown KE, Whitney SL, Wrisley DM, Furman JM. Physical therapy outcomes for persons with bilateral vestibular loss. Laryngoscope 2001; 111:1812-7

12. Shepard NT, Telian SA. Programmatic vestibular rehabilitation. Otolaryngol Head Neck Surg 1995; 112:173-82.

13. Herdman SJ, Blatt PJ, Schubert MC. Vestibular rehabilitation of patients with vestibular hypofunction or with benign paroxysmal positional vertigo. Curr Opinion Neurol 2000; 13:39-43.

14. Fife TD, Tusa RJ, Furman JM, Zee DS, Frohman E, Baloh RW et al. Assessment: vestibular testing techniques in adults and children: report of the therapeutics and technology assessment subcommittee of the American Academy of Neurology. Neurology 2000; 55(10):1431:41.

15. Kaplan DM, Marais J, Ogawa T, Kraus M, Rutka JA, Bance ML. Does high-frequency pseudo-random rotational chair testing increase the diagnostic yield of the electronystagmography caloric test in detecting bilateral vestibular loss in the dizzy patient? Laryngoscope 2001; 111:959-63.

16. Horak FB, Jones-Rycewicz C, Black FO, Shumway-Cook A. Effects of vestibular rehabilitation on dizziness and imbalance. Otolaryngol Head Neck Surg 1992; 106:175-80.

17. Zee DS. Vestibular adaptation. In: Herdman SJ. Vestibular Rehabilitation. $1^{\text {st }}$ ed. Philadelphia: F. A. Davis Company; 2000. p. 77-86.

18. Bles W, De Jong JMBV, De Wit G. Compensation for vestibular defects examined by the use of a tilting room. Acta Otolaryngol (Stockh) 1983; 95:576.

19. Clendaniel RA, Tucci DL. Vestibular rehabilitation strategies in Meniere's disease. Otolaryngol Clin North Am 1997; 30(6):1145-58.

20. Wiest G, Demer JL, Tian J, Crane BT, Baloh RW. Vestibular function in severe bilateral vestibulopathy. J Neurol Neurosurg Psychiatry 2001; 71:53-7. 